\title{
Higher Sensitivity Secondary Ion Mass Spectrometry of Biological Molecules for High Resolution, Chemically Specific Imaging
}

\author{
Liam A. McDonnell and Ron M. A. Heeren \\ FOM Institute for Atomic and Molecular Physics, Amsterdam, The Netherlands
}

Robert P. J. de Lange

Rudolph Magnus Institute of Neurosciences, Utrecht, The Netherlands

\author{
Ian W. Fletcher \\ ICI PLC, Measurement Science Group, Redcar, United Kingdom
}

\begin{abstract}
To expand the role of high spatial resolution secondary ion mass spectrometry (SIMS) in biological studies, numerous developments have been reported in recent years for enhancing the molecular ion yield of high mass molecules. These include both surface modification, including matrix-enhanced SIMS and metal-assisted SIMS, and polyatomic primary ions. Using rat brain tissue sections and a bismuth primary ion gun able to produce atomic and polyatomic primary ions, we report here how the sensitivity enhancements provided by these developments are additive. Combined surface modification and polyatomic primary ions provided $\approx 15.8$ times more signal than using atomic primary ions on the raw sample, whereas surface modification and polyatomic primary ions yield $\approx 3.8$ and $\approx 8.4$ times more signal. This higher sensitivity is used to generate chemically specific images of higher mass biomolecules using a single molecular ion peak. (J Am Soc Mass Spectrom 2006, 17, 1195-1202) (C) 2006 American Society for Mass Spectrometry
\end{abstract}

$\mathrm{T}$ The appeal of imaging mass spectrometry for studying biological samples is readily understandable. The heterogeneous structure of tissue and cells is apparent to even the most untrained eye. Furthermore, it has been established that the spatial distribution of biomolecules is intrinsically related to their role [1-3], and several diseases are associated with biomolecules having altered spatial distributions [4-6].

Chemical specificity is crucial in biological studies: the function of many biomolecules involves significant processing [7] and many ailments, whether hereditary, environmental, or pathological, are associated with modifications [6, 8]. The attraction of imaging mass spectrometry is the ability to map the concentration profiles of specific biomolecules, recording the distributions of multiple analytes in parallel, without using a label and from native samples (such as tissue sections).

Molecular imaging mass spectrometry is currently performed using either matrix assisted laser/desorption ionization (MALDI) or secondary ion mass spec-

Published online June 16, 2006

Address reprint requests to Professor R. M. A. Heeren, FOM Institute for Atomic and Molecular Physics, Kruislaan 407, 1098 SJ Amsterdam, The Netherlands. E-mail: heeren@amolf.nl trometry (SIMS) [9]. These two techniques offer different capabilities: MALDI is able to generate intact molecular ions of proteins in tissue [10]. However, to maintain high sensitivities for proteins, the spatial resolution of MALDI imaging is frequently $>50 \mu \mathrm{m}$. The other technique, SIMS, offers different capabilities: high-spatial resolution but a low mass range. SIMS is able to routinely provide sub-micron resolution images $[11,12]$, but its low mass range has meant that the analysis of larger molecules has been limited to those that can provide characteristic fragments. It has long been recognized that characteristic ions limit the application of SIMS, and much effort has focused on improving the sensitivity of SIMS for intact molecular ions of higher mass molecules. The most recent, and the subject of this paper, can be grouped into either surface modification or the use of polyatomic primary ions.

In SIMS imaging mass spectrometry, the impact of a highly focused primary ion beam with a surface sputters atoms and molecules from the top few $\mathrm{nm}$ of a surface. A small fraction of these species are ions; for molecules this is typically $<10^{-3}$ [12]. The ionization efficiency is strongly influenced by electron exchange processes between the departing species and the surface. This surface dependence of the ion yield is often 
Table 1. Experimental parameters of the SIMS experiments.

\begin{tabular}{|c|c|c|c|c|}
\hline & Spectroscopy & & & Imaging \\
\hline & $\mathrm{Bi}_{1}^{+}$ & $\mathrm{Bi}_{3}{ }^{2+}$ & $\ln _{1}{ }^{+}$ & $\mathrm{Bi}_{3}{ }^{2+}$ \\
\hline Ion current/pA & 0.5 & 0.09 & 0.009 & 0.009 \\
\hline Beam energy/kV & 20 & 20 & 15 & 20 \\
\hline Pulse length/ns & 0.84 & 0.85 & 20 & 100 \\
\hline Spot diameter/ $\mu \mathrm{m}$ & 4 & 4 & $\approx 0.7$ & $\approx 0.3$ \\
\hline Ion dose $/ \mathrm{cm}^{-2}$ & $4.14 \times 10^{12}$ & $4.14 \times 10^{12}$ & $1.7 \times 10^{12}$ & $2.35 \times 10^{11}$ \\
\hline Raster $/ \mu \mathrm{m}$ & 150 & 150 & 150 & 150 \\
\hline Pixels & $256 \times 256$ & $256 \times 256$ & $256 \times 256$ & $512 \times 512$ \\
\hline
\end{tabular}

referred to as matrix effects. The central role of the sample surface has led to the development of several sample preparation techniques that have demonstrated increased molecular ion yields of high mass molecules. These are: (1) matrix-enhanced SIMS (ME-SIMS) [1320] - uses the sample preparation protocols of MALDI to benefit from the detanglement [21] and chemical ionization aspects of MALDI [22-24]. Molecular ions of analytes as large as ubiquitin and lysozyme have been detected, and for lower mass analytes, $<2 \mathrm{kDa}$, the ME-SIMS spectra frequently resemble those obtained using MALDI [17, 19]. ME-SIMS has increased the sensitivity for lower mass macromolecules such that subcellular distributions of cholesterol and small peptides have been recorded, using their chemical-specific molecular ions $[19,20]$. The addition of salts and acids to the sample surface has also provided increased yields for analytes known to bind metal cations and protons, respectively [25].

(2) Sample metallization-applying a thin-layer of gold or silver to the sample has been shown to provide increased sensitivities for large analytes as diverse as polymers, dyes, pharmaceuticals, fatty acids, lipids, and cholesterol [26-29]. The mechanism behind this increased sensitivity has been attributed to analyte migration onto the nano-islands produced by the deposition, the increased stopping power of gold (thus more energy remains in the surface region), and to the catalytic properties of these nano-islands [27]. Nygren et al. have exploited sample metallization to record the distribution of cholesterol in a rat kidney tissue section of a Male Sprague-Dawley, which revealed a high concentration in the nuclear region of epithelial cells. [29] In this case the cholesterol image was obtained using the silver cationized molecular ions.

Without application of the matrix or the thin metal coatings, such high-resolution images are not possible because the sensitivity is insufficient. The other technique that has been developed for improving the sensitivity of high mass molecules is polyatomic primary ions. Enhanced sputtering by polyatomic primary ions has been known for more than four decades. In 1960, the groups of Kistemaker [30] and Moore [31] independently demonstrated that polyatomic projectiles sputter more material than that expected from independent atomic projectiles. It has been demonstrated that these enhanced yields are produced with a concomitant in- crease in surface damage, but the yield enhancement surpasses that of damage [32]. Many studies have combined to develop the now accepted idea that the lower penetration depth and overlapping collision cascades of the polyatomic primary ion deposit more energy in the surface region, thus leading to increased sputtering [33-39].

The new developments in polyatomic primary ions have been making these sensitivity enhancements compatible with imaging mass spectrometry by designing ion sources and ion optics that can provide the focused ion beam necessary for high-resolution microprobe imaging. These are the gold [40] and bismuth [41] cluster guns, and the $\mathrm{C} 60+$ gun developed by Vickerman and workers [42, 43]. The enhanced sputtering by the latter polyatomic primary ion has been explicitly demonstrated: using a quartz-crystal microbalance it was estimated that, on average, each impact of $\mathrm{C} 60$ ions on a frozen sample sputtered $\approx 2400$ water molecules [44].

Following the preposition that surface modification and polyatomic primary ions can enhance different aspects of the SIMS process, namely ionization and sputtering respectively, here we have addressed the question of whether their sensitivity gains are additive: whether one can increase the molecular ion yield of high mass molecules even further by combining both techniques.

\section{Experimental}

The experiments were performed using either a TRIFT-II (Physical Electronics, Eden Prairie, MN) equipped with an indium liquid metal ion gun and a nitrogen laser (for MALDI), or a TOF-SIMS IV (IONTOF $\mathrm{GmbH}$, Münster, Germany) equipped with a Bismuth ion gun that is capable of producing $\mathrm{Bi}_{1}^{+}, \mathrm{Bi}_{3}^{+}$, and $\mathrm{Bi}_{3}^{2+}$ with sufficient intensity for practical imaging experiments. Table 1 shows the experimental parameters used for these experiments. All SIMS experiments were well within the static SIMS limit [12] (statistically a fresh area is sampled with each primary ion impact), thus ensuring that the spectra are representative of the sample surface. The ten $\mu \mathrm{m}$ thick rat brain tissue sections were cut at interaural 7.2 /bregma $1.8 \mathrm{~mm}$ [45] using a cryomicrotome and thaw mounted on indium-tin-oxide slides (conductive, transparent slides). These were then placed in custom-made stainless steel sample holders 

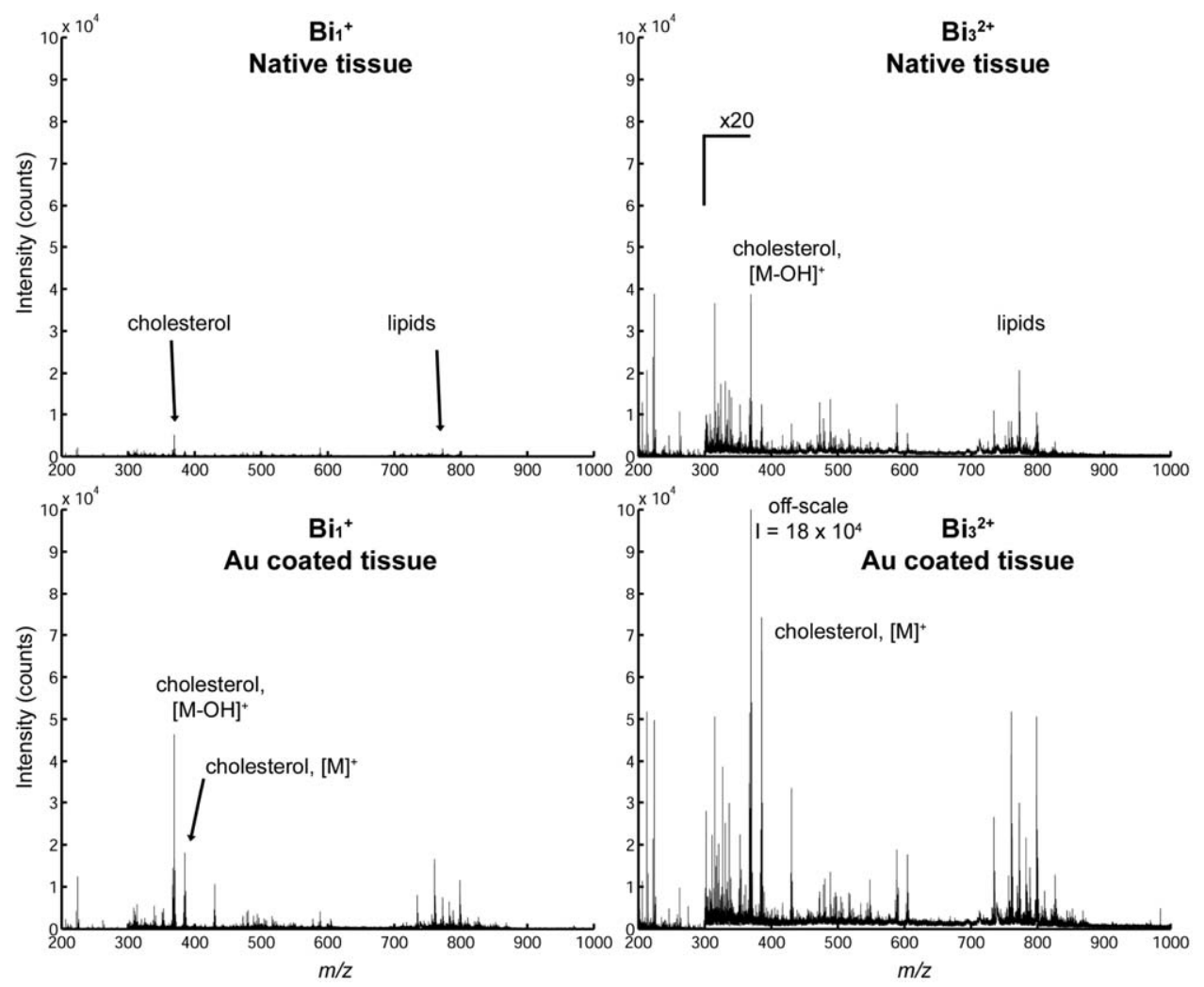

Figure 1. Comparison of the positive-ion SIMS spectra obtained from uncoated and gold-coated tissue sections using $\mathrm{Bi}_{1}^{+}$and $\mathrm{Bi}_{3}^{2+}$ primary ions. The mass scales and intensity scales are identical to aid comparison. Primary ion dose $=4.12 \times 10^{12} \mathrm{~cm}^{-2}$.

and stored at $-80^{\circ} \mathrm{C}$ until required. The rats, $350 \mathrm{~g}$ male Wistar rats (Crl:WU) obtained from Charles River (Sulzfeld, Germany), were decapitated without prior anesthesia, the brains dissected and flash frozen in liquid isopentane, and then stored at $-80^{\circ} \mathrm{C}$ until sectioning. Experimental procedures were in accordance with the European directives (86/609/EEC) and approved by the Commission on Laboratory Animal Experiments of the Utrecht University Medical Center.

The gold-coated samples were prepared by depositing a $1 \mathrm{~nm}$ gold layer on top of the tissue sections using a sputter coater (SC7640 Quorum Technologies, Newhaven, UK). The ME-SIMS samples were prepared using a pneumatic sprayer to deposit a thin-layer of matrix, 2,5-dihydroxybenzoicacid (DHB), onto the tissue sections. All data analyses and visualization was performed using user-programs written for Matlab version 6.5 .

\section{Results and Discussion}

The results shown here compare the performance of surface modifications and polyatomic primary ions for SIMS imaging of rat brain tissue sections. Gold coated tissue sections, matrix coated tissue-sections, and native (uncoated) tissue sections were analyzed using $\mathrm{Bi}_{1}^{+}$and $\mathrm{Bi}_{3}^{2+}$ primary ions in positive and negative ion mode. Figure 1 compares the positive ion spectra obtained from native and gold-coated tissue sections. To emphasize differences in intensity, the scales of all four spectra are identical. The top row shows the spectra from native, uncoated tissue sections. It is readily apparent that, as seen in previous studies, the $\mathrm{Bi}_{3}^{2+}$ primary ion generates significantly greater molecular-ion signals: cholesterol, diacylglycerols, and lipids are all clearly observed. The $\mathrm{Bi}_{3}^{2+}$ primary ions delivered approximately an order of magnitude more molecular ion signal than the $\mathrm{Bi}_{1}^{+}$primary ions.

The use of $\mathrm{Bi}_{1}^{+}$primary ions and a thin gold coating, bottom left spectrum, produces a spectrum that appears comparable to that obtained with $\mathrm{Bi}_{3}^{2+}$ from native tissue: similar intensities and similar spectral features. The final spectrum in Figure 1, bottom right, shows that the intensity gains offered by polyatomic primary ions and gold coating the sample are approximately additive. This combination yielded the most intense peaks for all molecular ion classes observed: cholesterol (off scale, intensity $=18 \times 10^{4}$ counts) and lipids. Compared with the SIMS results of the uncoated sample using atomic $\mathrm{Bi}^{+}$primary ions, the lipid peaks are $\sim 16$ times more intense. From an imaging perspective, these increased yields provide significantly greater contrast or can permit four times higher spatial resolution at the same contrast.

Closer examination of the lipid region revealed subtle changes. Figure 2 shows a close-up in which the 

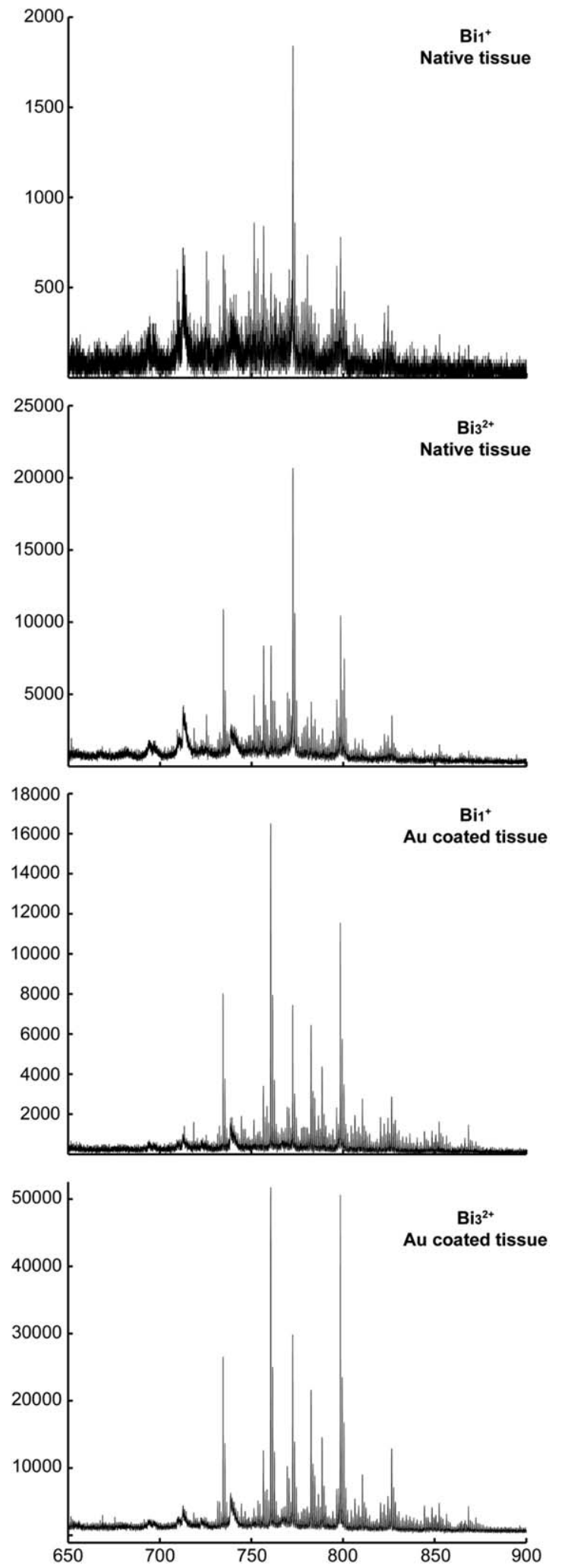

Figure 2. Close-up of the lipid molecular ion region of Figure 1. Intensity scales optimized for spectral clarity.

vertical scale has been optimized for spectral clarity. For the native tissue, the $\mathrm{Bi}_{3}^{2+}$ primary ion generates the same molecular ions as the atomic $\mathrm{Bi}_{1}^{+}$primary ion, but with significantly greater yield. When the sample was prepared by applying a thin gold coating before analysis, their relative intensities changed, although the $\mathrm{m} / \mathrm{z}$ of the major peaks remained constant. These "altered" relative intensities were generated in both positive-ion gold-coated experiments, for $\mathrm{Bi}_{1}^{+}$and $\mathrm{Bi}_{3}^{2+}$ primary ions. Nevertheless, the major peaks from the native samples were reproduced in the gold-coated sample with increased intensity. The cause of the change in relative intensities requires further investigation.

Figure 2 also shows that, independent of sample preparation and primary ion, each spectrum obtained from the tissue sections contains broad, low mass resolution peaks. For example, in the $\mathrm{Bi}_{3}^{2+}$ spectrum from native tissue broad peaks can be seen at $m / z 695$, 711, 714, and 739. As with the regular, higher mass resolution peaks, the relative intensities of these broad peaks also changes when a gold coating is applied. Such low-resolution peaks are normally attributed to metastable decay.

The benefits of polyatomic primary ions and goldcoating the sample are less striking in the negative ion spectra. Figure 3 shows the negative ion spectra obtained from native and gold-coated tissue sections using $\mathrm{Bi}_{1}^{+}$and $\mathrm{Bi}_{3}^{2+}$ primary ions. On native tissue, the use of the polyatomic primary ion resulted in more modest improvements except for the highest $\mathrm{m} / \mathrm{z}$ peaks, where the increase in molecular ion yield was comparable to that seen in positive ion mode. $\mathrm{Bi}_{1}^{+}$on the gold-coated sample increases the yield of many of the molecular ions seen with the native tissue but also generates new peaks, the most intense of which are clusters of gold, sulfur, and hydrogen (labeled with an asterisk $^{*}$ ), which can be used as for accurate mass calibration.

Surprisingly, polyatomic primary ions combined with gold coating did not provide the most intense signals, as seen in positive ion mode. Although the more intense peaks of the other spectra were also present in this spectrum (labeled \# in the $\mathrm{Bi}_{3}^{2+}$ spectra), their intensities were less. Note that owing to severe time restrictions, this spectrum was recorded with approximately half the primary ion dose of the other spectra. However, as all ions displayed identical, and the expected, decay profiles and all spectra were recorded within the static SIMS limit, this was not the cause of their lower ion yields. This unexpected result indicates one of the disadvantages of gold coating, the difficulty in predicting which peaks will be enhanced. Nevertheless, the enhanced signal intensities reported for positive ion mode, and particularly when combined with polyatomic primary ions, remain an invaluable addition for a technique bedeviled by low macromolecular ion signal intensities.

Figure 4 summarizes the relative sensitivity improvements for higher mass molecular ions (greater than $\mathrm{m} / \mathrm{z} 600$ ) using surface modifications, polyatomic primary ions, and their combination on rat brain tissue sections. Clearly, the molecular ion yield improvements 

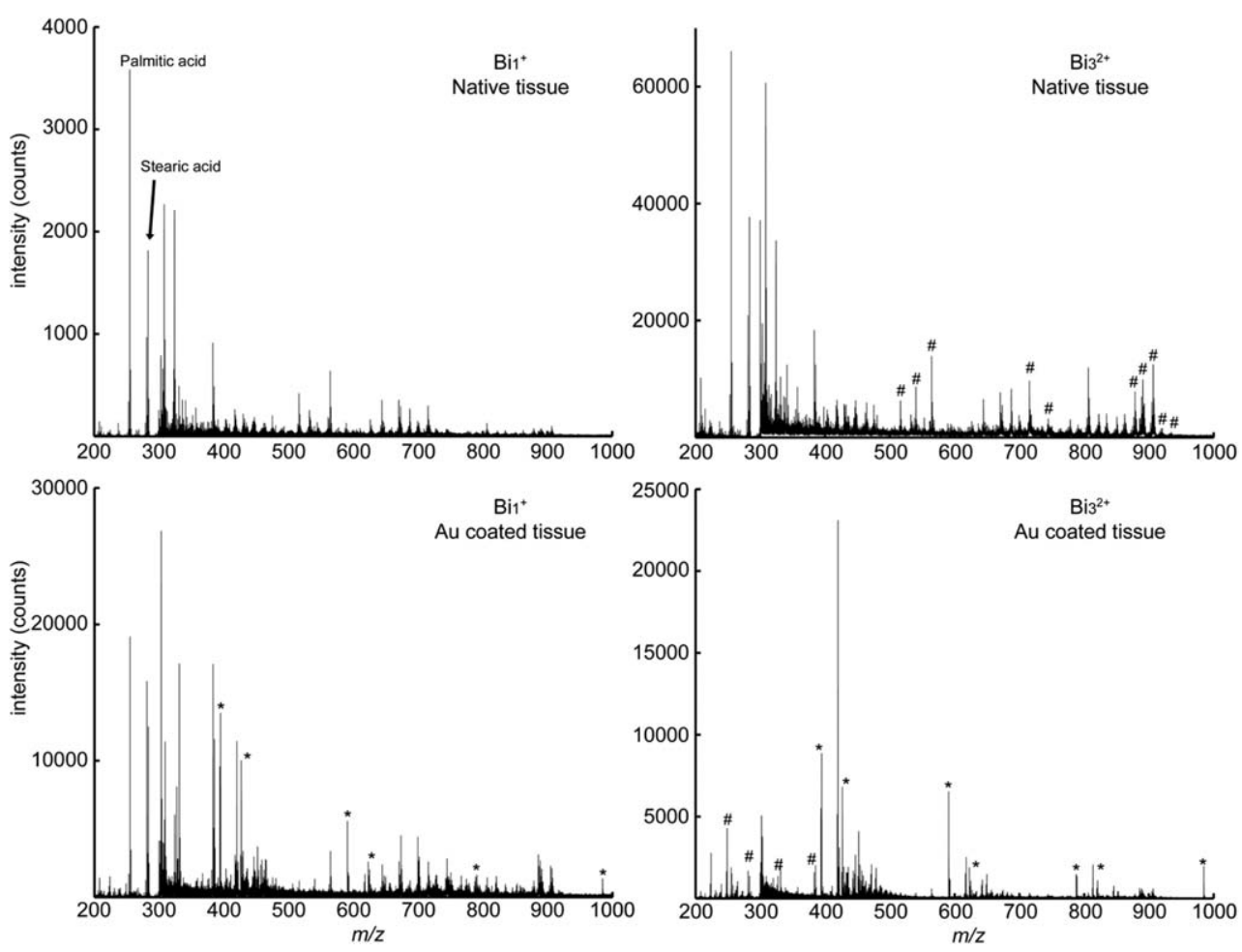

Figure 3. Comparison of the negative-ion SIMS spectra obtained from uncoated and gold-coated tissue sections using $\mathrm{Bi}_{1}^{+}$and $\mathrm{Bi}_{3}^{2+}$ primary ions.

delivered by surface modification and polyatomic primary ions are additive, and the most intense signals are obtained by exploiting their combination. Additionally, these ME-SIMS results were obtained from the negative ion spectra, thus demonstrating that the additivity can be in both positive and negative ion spectra. The unexpected negative ion gold coating results were

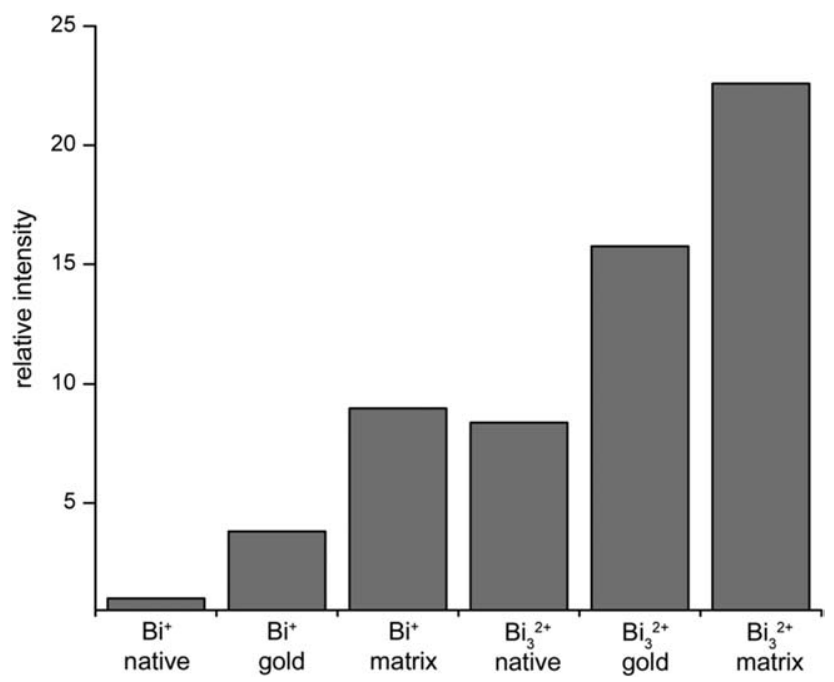

Figure 4. Comparison of ion yield enhancements of the higher mass molecular ions obtained with $\mathrm{Bi}_{3}^{2+}$ polyatomic primary ions (with respect to $\mathrm{Bi}_{1}^{+}$atomic primary ions) for uncoated, gold coated, and matrix coated tissue sections. included for completion, to demonstrate the significant advantages but also to guard against the complexities.

Polyatomic primary ions, Au-SIMS and ME-SIMS, have all been developed to increase the sensitivity of SIMS for higher mass analytes, to expand the high spatial resolution capabilities of SIMS imaging to these higher mass analytes. Consequently, any surface modification technique must be applied in a manner that does not disturb the spatial distribution within the length scale of the imaging experiment (typically $1 \mu \mathrm{m}$ ). Gold coating has been shown to result in nanometer scale gold islands, while electrospray matrix depositions demonstrated experimental spatial resolution of tissue sections of $2.9 \mu \mathrm{m}$ [20]. This example concerns 2,5-dihydroxybenzoic acid but the technique is identical for application of salts and acids.

\section{Imaging}

Figure 5 demonstrates the benefit of combining polyatomic primary ions and surface modification. It shows the results of a large-scale, high spatial resolution imaging experiment of a gold-coated rat brain tissue section using $\mathrm{Bi}_{3}^{2+}$ primary ions (the combination that provided the highest molecular signals in Figure 1). An unbunched primary ion beam was used to ensure high spatial resolution, but at the expense of mass resolution. Nevertheless, the mass spectrum clearly includes the same peaks as in Figure 1, and that molecular ions of cholesterol and lipids are produced with sufficient 


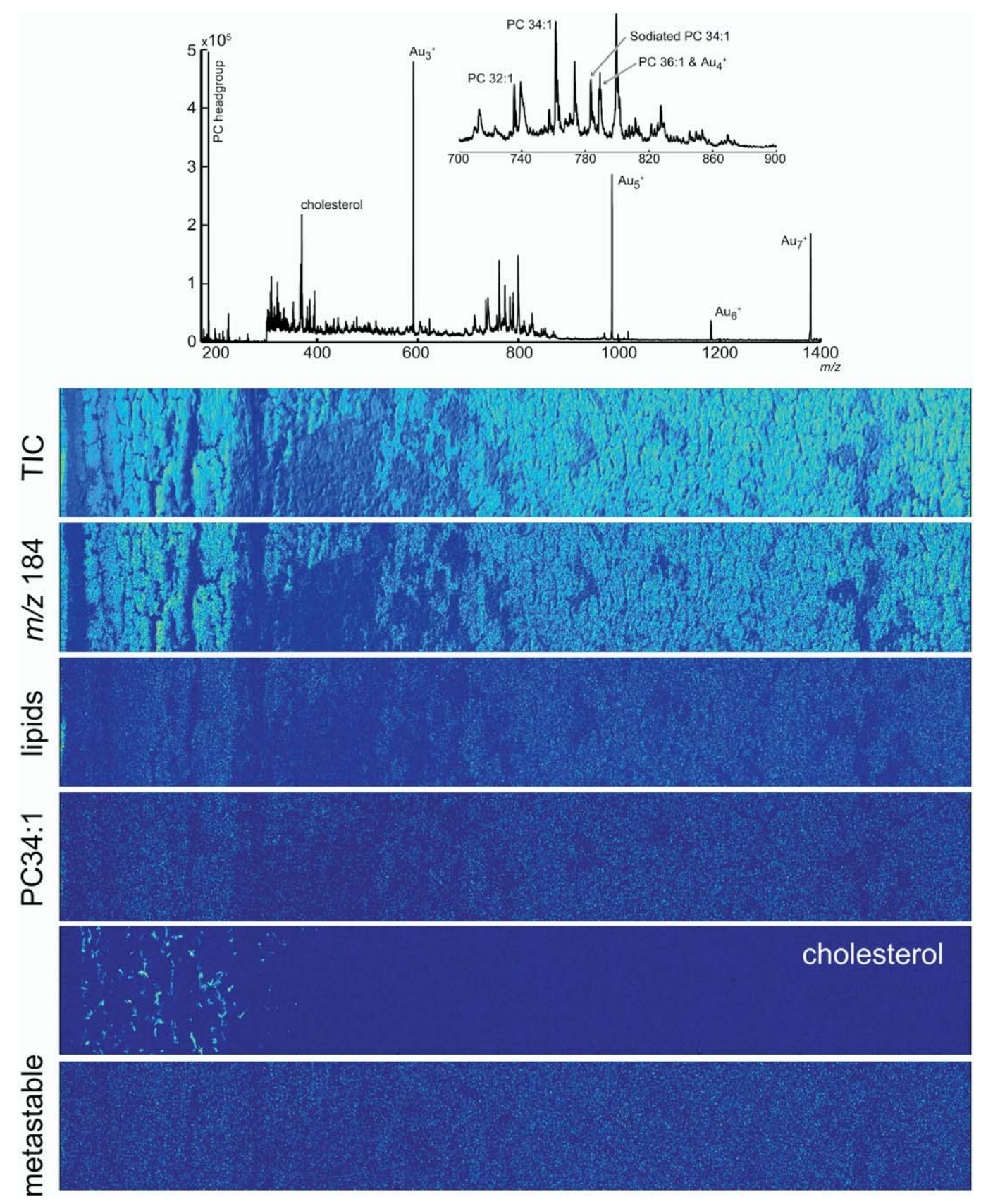

Figure 5. Large scale, high spatial resolution imaging experiment of a gold-coated rat brain tissue section using $\mathrm{Bi}_{3}^{2+}$ primary ions, detecting positive secondary ions. The spectrum shows the signals obtained from the entire analysis area, including some assignments. The total-ion-count (TIC) image is included to clearly distinguish the cellular features of the tissue section. The spatial distribution of the phosphocholine headgroup $\left(\mathrm{PO}_{4} \mathrm{NC}_{5} \mathrm{H}_{15}^{+}, \mathrm{m} / z\right.$ 184), the entire lipid region $(\mathrm{m} / \mathrm{z} 710-825)$, the specific lipid PC 34:1 obtained from just its protonated molecular ion, cholesterol and the metastable peak $m / z \approx 739$ are shown in the following images. To improve contrast the phosphocholine headgroup, lipid region, PC34:1, cholesterol and metastable images were rebinned: the intensity of 3 $\times 3$ pixels were summed to create a single pixel in the new image.

intensity for high-resolution imaging. The total-ioncurrent (TIC) image, top image, has been included to distinguish the cellular features of the tissue section. The next three images show the spatial distribution of the phosphocholine headgroup $(\mathrm{m} / \mathrm{z} 184)$, the lipid envelope $(m / z 710-825)$, and the single phospholipid phosphatidylcholine 34:1 at $\mathrm{m} / \mathrm{z} 761$.
The intense phosphocholine headgroup fragment is often used to record high-resolution images of phosphatidylcholine (PC) or phospholipids in general. However, it is unspecific in both cases: sphingomyelin also generates the $m / z 184$ fragment and, while it is the most common lipid in the brain, is just one of many lipids. In a previous report of the sensitivity improvements of- 
fered by gold and bismuth cluster primary ions, images have been presented of the molecular ions contained in the entire lipid envelope, (which also imaged large areas using a large pixel size) [41]. While this provides more signal intensity, it is nonspecific. The increased molecular ion yields offered by combining surface modification and polyatomic primary ions provide sufficient intensity for imaging specific molecular ions of lipids. The fourth image shows the distribution of PC34:1 using just the protonated molecular ion. Cellular features can be clearly distinguished in this high-mass, chemical-specific image. Moreover, these images were recorded with a low primary ion dose, 20 times less than those used to record the spectra of Figures 1-3. With higher primary ion currents or a faster repetition rate, high contrast chemical-specific images could be recorded (it was not practical to record the image with the same ion dose as Figure 1-3: it would have taken $\approx 60 \mathrm{~h}$ ). The final images show the distribution of cholesterol and the metastable peak at $m / z$ 739. The cholesterol image shows some higher intensity regions near the edge of the tissue, and the metastable peak is almost uniformly distributed.

The results shown above demonstrate that the yield enhancements provided by polyatomic primary ions and surface modifications can be additive, and that they can be exploited to obtain high-resolution images of specific molecular ions. The unexpected negative ion result, the lower intensity of the combination of gold coating, and polyatomic primary ions showed the disadvantage: despite several reports utilizing thin metal coatings for increased sensitivity, and one even claiming that it reduces matrix effects in SIMS [46], it can be difficult to predict which systems, under which conditions, benefit from metal coatings. This raises the question of when an analyst should use surface modifications. Clearly, the numerous laboratories that do not have access to the latest, and expensive, polyatomic primary ion beams will benefit from the use of gold coating, or the application of matrices, metal salts, or acids, the choice of sample preparation being dependent on the analyte of interest. For the laboratories that possess gold, bismuth, or $\mathrm{C}_{60}$ primary ion guns, it is the analyst's choice whether to exploit a demonstrated yield improvement of up to $150 \%$ (Figure 1). For a field that has been limited by low ion yields of intact high mass molecules, and in which the multivariate tools used to extract diagnostic information from the complex, fragment-rich spectra perform better with increased signal intensity [47], many analysts will exploit whatever techniques provide increased performance.

\section{Conclusions}

The improvements in sensitivity for molecular ions of high mass analytes that can be provided by polyatomic primary ions and surface modification are additive. With respect to monatomic $\mathrm{Bi}_{1}^{+}$primary ions examining an uncoated tissue section, $\mathrm{Bi}_{1}^{+}$on a gold-coated tissue section provided $\approx 3.8$ higher molecular ion lipid signals, $\mathrm{Bi}_{3}^{2+}$ on uncoated tissue provided $\approx 8.4$ more molecular ion lipid signals, and $\mathrm{Bi}_{3}^{2+}$ on gold-coated tissue provided the most signal, $\approx 15.8$ times as much signal. This higher sensitivity can be used to provide chemically specific images of higher mass biomolecules using a single molecular ion peak. The spectra obtained from the gold-coated tissue section also illustrate the complex effects of this sample preparation; specifically the difficulty in predicting which ions will be generated with increased sensitivity. Nevertheless, the results shown here and the wide range of compounds already shown to be consistent with gold coating demonstrate the utility of this technique, and it is debatable if these matrix effects are more or less complex than those of examining uncoated tissue sections.

\section{Acknowledgments}

This work is part of research program no. 49, "Mass spectrometric imaging and structural analysis of biomacromolecules" of the "Stichting voor Fundamenteel Onderzoek der Materie (FOM)," which is financially supported by the Nederlandse organisatie voor Wetenschappelijk Onderzoek (NWO).

\section{References}

1. Dreger, M. Subcellular Proteomics. Mass Spectrom. Rev. 2003, 22, 27-56.

2. Kumar, A.; Agarwal, S.; Heyman, J. A.; Matson, S.; Heidtman, M. Piccirillo, S.; Umansky, L.; Drawid, A.; Jansen, R.; Liu, Y.; Cheung, K.-H.; Miller, P.; Gerstein, M.; Roeder, G. S.; Snyder, M. Subcellular Localization of the Yeast Proteome. Genes Dev. 2002, 16, 707-719.

3. Simpson, J. C.; Wellenreuther, R.; Poustka, A.; Pepperkok, R.; Wiemann, S. Systematic Subcellular Localization of Nnovel Proteins Identified by Large-Scale cDNA Sequencing. EMBO Reports 2000, 1, 287-292.

4. Roher, A. E.; Weiss, N.; Kokjohn, T. A.; Kuo, Y. M.; Kalback, W.; Anthony, J.; Watson, D.; Luehrs, D. C.; Sue, L.; Walker, D.; Emmerling, M.; Goux, W.; Beach, T. Increased A $\beta$ Peptides and Reduced Cholesterol and Myelin Proteins Characterize White Matter Degeneration in Alzheimer's Disease. Biochem. 2002, 41, 11080-11090.

5. Soderberg, M.; Edlund, C.; Alafuzoff, I.; Kristensson, K.; Dallner, G. Lipid Composition in Different Regions of the Brain in Alzheimer's Disease Senile Dementia of Alzheimer's Type. J. Neurochem. 1992, 59, 1646-1653.

6. Hanash, S. Disease Proteomics. Nature 2003, 422, 226-232.

7. Tyndall, J. D. A.; Nall, T.; Fairlie, D. P. Proteases Universally Recognize $\beta$ Strands in Their Active Sites. Chem. Rev. 2005, 105, 973-999.

8. Xia, G. G. S.; Nel, A. E.; Loo, J. A. Nitrotyrosine-Modified Proteins and Oxidative Stress Induced by Diesel Exhaust Particles. Electrophoresis 2005, 26, 280-292.

9. Todd, P. J.; Schaaff, T. G.; Chaurand, P.; Caprioli, R. M. Organic Ion Imaging of Biological Tissue with Secondary Ion Mass Spectrometry and Matrix-Assisted Laser Desorption/Ionization. J. Mass Spectrom. 2001, 36, 355-369.

10. Chaurand, P.; Schwartz, S. A.; Caprioli, R. M. Profiling and Imaging Proteins in Tissue Sections by MS. Anal. Chem. 2004, 76, 86A-93A

11. Chabala, J. M.; Soni, K. K.; Li, J.; Gavrilov, K. L.; Levi-Setti, R. High Resolution Chemical Imaging with Scanning Ion Probe SIMS. Int. J. Mass Spectrom. 1995, 143, 191-212.

12. Vickerman, J. C.; Briggs, D., Eds. TOF-SIMS: Surface Analysis by Mass Spectrometry; IM Publications: Chichester, 2001, p. 417.

13. Nicola, A. J.; Muddiman, D. C.; Hercules, D. M. Enhancement of Ion Intensity in Time-of-Flight Secondary-Ionization Mass Spectrometry. J. Am. Soc. Mass Spectrom. 1996, 7, 467-472.

14. Wu, K. J.; Odom, R. W. Matrix-Enhanced Secondary Ion Mass Spectrometry: A Method for Molecular Analysis of Solid Surfaces. Anal. Chem. 1996, 68, 873-882.

15. Hanton, S. D.; Cornelio Clark, P. A.; Owens, K. G. Investigations of Matrix-Assisted Laser Desorption/Ionization Sample Preparation by Time-of-Flight Secondary Ion Mass Spectrometry. J. Am. Soc. Mass Spectrom. 1999, 10, 104-111.

16. Wittmaack, K.; Szymczak, W.; Hoheisel, G.; Tuszynski, W. Time-ofFlight Secondary Ion Mass Spectrometry of Matrix-Diluted Oligon- and Polypeptides Bombarded with Slow and Fast Projectiles: Positive and Negative Matrix and Analyte Ion Yields, Background Signals, and Sample Aging. J. Am. Soc. Mass Spectrom. 2000, 11, 553-563. 
17. Luxembourg, S. L.; McDonnell, L. A.; Duursma, M.; Guo, X.; Heeren R. M. A. Effect of Local Matrix Crystal Variations in Matrix-Assisted Ionization Techniques for Mass Spectrometry. Anal. Chem. 2003, 75, 2333-2341.

18. McDonnell, L. A.; Luxembourg, S. L.; Mize, T. H.; Koster, S.; Eijkel, G. B.; Verpoorte, E.; de Rooij, N. F.; Heeren, R. M. A. Using Matrix Peak to Map Topography: Increased Mass Resolution and Enhanced Sensitivity in Chemical Imaging. Anal. Chem. 2003, 75, 4373-4381.

19. McDonnell, L. A.; Piersma, S. R.; Altelaar, A. F. M.; Mize, T. H. Luxembourg, S. L.; Verhaert, P. D. E. M.; van Minnen, J.; Heeren, R. M. A. Subcellular Imaging Mass Spectrometry of Brain Tissue. J. Mass Spectrom. 2005, 40, 160-168.

20. Altelaar, A. F. M.; van Minnen, J.; Jiménez, C. R.; Heeren, R. M. A.; Piersma, S. R. Direct Molecular Imaging of Lymnaea stagnalis Nervous Tissue at Subcellular Spatial Resolution by Mass Spectrometry. Anal. Chem. 2005, 77, 735-741.

21. Delcorte, A.; Garrison, B. J. Particle-Induced Desorption of Kilodalton Molecules Embedded in a Matrix: A Molecular Dynamics Study. J. Phys. Chem. B. 2003, 107, 2297-2310.

22. Knochenmuss, R.; Zenobi, R. MALDI Ionization: The Role of In-Plume Processes. Chem. Rev. 2003, 103, 441-452.

23. Knochenmuss, R. A Quantitative Model of Ultraviolet Matrix-Assisted Laser Desorption/Ionization Including Analyte Ion Generation. Anal. Chem. 2003, 75, 2199-2207.

24. Breuker, K.; Knochenmuss, R.; Zhang, J.; Stortelder, A.; Zenobi, R Thermodynamic Control of Final Ion Distributions in MALDI: In-Plume Proton Transfer Reactions. Int. J. Mass Spectrom. 2003, 226, 211-222.

25. Delcorte, A.; Bertrand, P. Metal Salts for Molecular Ion Yield Enhancement in Organic Secondary Ion Mass Spectrometry: A Critical Assessment. Anal. Chem. 2005, 77, 2107-2115.

26. Altelaar, A. F. M., Klinkert, I., Jalink, K., de Lange, R. P. J., Adan, R. A. H., Heeren, R. M. A., Piersma, S. R. Gold-Enhanced Biomolecular Surface Imaging of Cells and Tissue by SIMS and MALDI Mass Spectrometry. Unpublished.

27. Delcorte, A.; Bour, J.; Aubriet, F.; Muller, J.-F.; Bertrand, P. Sample Metallization for Performance Improvement in Desorption/Ionization of Kilodalton Molecules: Quantitative Evaluation, Imaging Secondary Ion MS, and Laser Ablation. Anal. Chem. 2003, 75, 6875-6885.

28. Keune, K.; Boon, J. J. Enhancement of the Static SIMS Secondary Ion Yields of Lipid Moieties by Ultrathin Gold Coating of Aged Oil Paint Surfaces. Surf. Interface Anal. 2004, 36, 1620-1628.

29. Nygren, H.; Malmberg, P.; Kriegeskotte, C.; Arlinghaus, H. F. Bioimaging TOF-SIMS: Localization of Cholesterol in Rat Kidney Sections. FEBS Lett. 2004, 566, 291-293.

30. Rol, P. K.; Fluit, J. M.; Kistemaker, J. Sputtering of Copper by Bombardment with Ions of 5-25 KeV. Physica 1960, 26, 1000-1008.

31. Grøonlund, F.; Moore, W. J. Sputtering of Silver by Light Ions with Energies from 2 to $12 \mathrm{keV}$. J. Chem. Phys. 1960, 32, 1540-1545.

32. Appelhans, A. D.; Delmore, J. E. Comparison of Polyatomic and Atomic Primary Beams for Secondary Ion Mass Spectrometry of Organics. Anal. Chem. 1989, 61, 1087-1093.
33. Blain, M. G.; Della-Negra, S.; Joret, H.; Le Beyec, Y.; Schweikert, E. A. Secondary-Ion Yields from Surfaces Bombarded with keV Molecular and Cluster Ions. Phys. Rev. Lett. 1989, 63, 1625-1628.

34. Blain, M. G.; Della-Negra, S.; Joret, H.; Le Beyec, Y.; Schweikert, E. A. Desorption Yields Using KeV Polyatomic Projectiles. J. Phys. Paris 1989, 50, 147-153.

35. Blain, M. G.; Della-Negra, S.; Joret, H.; Le Beyec, Y.; Schweikert, E. A. A New Experimental Method for Determining Ion Yyields from Surfaces Bombarded by Complex Heterogeneous Ions. J. Vac. Sci. Technol. 1990, $8,2265-2268$.

36. Postawa, Z.; Czerwinski, B.; Szewczyk, M.; Smiley, E. J.; Winograd, N.; Garrison, B. J. Enhancement of Sputtering Yields Due to $C_{60}$ versus Ga Bombardment of $\mathrm{Ag}(111)$ as Explored by Molecular Dynamics Simulations. Anal. Chem. 2003, 75, 4402-4407.

37. Postawa, Z.; Czerwinski, B.; Szewczyk, M.; Smiley, E. J.; Winograd, N.; Garrison, B. J. Microscopic Insights into the Sputtering of $\mathrm{Ag}(111)$ Induced by $\mathrm{C}_{60}$ and Ga Bombardment. J. Phys. Chem. B 2004, 108, 7831-7838.

38. Nguyen, T. C.; Ward, D. W.; Townes, J. A.; White, A. K.; Krantzman, K. D. A Theoretical Investigation of the Yield-to-Damage Enhancement with Polyatomic Projectiles in Organic SIMS. J. Phys. Chem. B 2000, 107, 8221-8228.

39. Townes, J. A.; White, A. K.; Wiggins, E. N.; Krantzman, K. D.; Garrison, B. J.; Winograd, N. Mechanism for Increased Yield with SF $_{5}^{+}$Projectiles in Organic SIMS: The Substrate Effect. J. Phys. Chem. A 1999, 103, 4587-4589.

40. Benguerba, M.; Brunelle, A.; Della-Negra, S.; Depauw, J.; Joret, H.; Le Beyec, Y.; Blain, M. G.; Schweikert, E. A.; Assayag, G. B.; Sudraud, P. Impact of Slow Gold Clusters on Various Solids: Nonlinear Effects in Secondary Ion Emission. Nucl. Instrum. Methods Phys. Res. B 1991, 62, $8-22$.

41. Touboul, D.; Kollmer, F.; Niehuis, E.; Brunelle, A.; Laprévote, O. Improvement of Biological Time-of-Flight Secondary Ion Mass Spectrometry Imaging with Bismuth Cluster Ion Source. J. Am. Soc. Mass Spectrom. 2005, 16, 1608-1618.

42. Wong, S. C. C.; Hill, R.; Blenkinsopp, P.; Lockyer, N. P.; Weibel, D. E.; Vickerman, J. C. Development of a C-60(+) Ion Gun for Static SIMS and Chemical Imaging. Appl. Surf. Sci. 2003, 203, 219-222.

43. Weibel, D.; Wong, S.; Lockyer, N.; Blenkinsopp, P.; Hill, R.; Vickerman, J. C. A $C_{60}$ Primary Ion Beam System for Time of Flight Secondary Ion Mass Spectrometry: Its Development and Secondary Ion Yield Characteristics. Anal. Chem. 2003, 75, 1754-1764.

44. Wucher, A.; Sun, S.; Szakal, C.; Winograd, N. Molecular Depth Profiling of Histamine in Ice Using a Buckminster Fullerene Probe. Anal. Chem. 2004, 76, 7234-7242.

45. Paxinos, G.; Watson, C. The Rat Brain in Stereotaxic Coordinates; Academic Press: Orlando, 1998, p. 26.

46. Inoue, M.; Murase, A. Reduction of Matrix Effects in TOF-SIMS Analysis by Metal-Assisted SIMS (META-SIMS). Surf. Interface Anal. 2005, in press.

47. Wickes, B. T.; Kim, Y.; Castner, D. G. Denoizing and Multivariate Analysis of Time-of-Flight SIMS Images. Surf. Interface Anal. 2003, 35, $640-648$. 